\title{
Resistividade do filme depositado via sol-gel e estado de oxidação do dopante Ce na matriz $\mathrm{SnO}_{2}$
}

\author{
(Resistivity of the film deposited via sol-gel and oxidation state \\ of Ce doping in $\mathrm{SnO}_{2}$ matrix)
}

\author{
L.V.A.Scalvi ${ }^{1,2}$, T.F.Pineiz ${ }^{3}$, M.A.L.Pinheiro ${ }^{3}$, M.J.Saeki ${ }^{2,4}$, V. Briois ${ }^{5}$ \\ ${ }^{I}$ Dep. Física - FC, ${ }^{2}$ Grupo de Materiais Avançados (MAV), ${ }^{3}$ Programa de Pós-Graduação em Ciência e \\ Tecnologia Materiais - POSMAT, ${ }^{4}$ Dep. Química e Bioquímica, Instituto de Biociências \\ Universidade Estadual Paulista - UNESP, C.P. 473, Bauru, SP 17033-360, Brasil \\ ${ }^{5}$ SOLEIL Synchrotron - L'Orme des Merisiers, Gif sur Yvette, França
}

\begin{abstract}
Resumo
Incorporação de $\mathrm{Ce}^{3+}$ ou $\mathrm{Ce}^{4+}$ em filmes finos de $\mathrm{SnO}_{2}$ depositados via sol-gel-dip-coating aumenta drasticamente a resistividade elétrica. No primeiro caso, temos comportamento aceitador do dopante, levando a matriz à alta compensação de carga. Por outro lado, para $\mathrm{Ce}^{4+}$, verifica-se aumento na largura da região de depleção do contorno de grão, resultando em maior espalhamento de elétrons. Medidas de caracterização elétrica sob pressão ambiente levam à barreiras de potencial mais altas do que as medidas sob vácuo, devido a adsorção de oxigênio na superfície das partículas. A presença de $\mathrm{Ce}^{3+}$ aumenta a transmitância no infravermelho,o que significa menor quantidade de elétrons livres. Dados de XANES confirmam que o tratamento térmico a $550{ }^{\circ} \mathrm{C}$ dos filmes, ainda que promova oxidação parcial para $\mathrm{Ce}^{4+}$, preserva uma quantidade significativa (em torno de $60 \%$ ) no estado $\mathrm{Ce}^{3+}$. Espectroscopia Raman mostra a evolução dos modos de vibração intra-grãos de $\mathrm{SnO}_{2}$ com o aumento da temperatura de tratamento térmico.

Palavras-chave: dióxido de estanho, filmes finos, cério, terras-raras, transporte elétrico.
\end{abstract}

\begin{abstract}
Incorporation of $\mathrm{Ce}^{3+}$ or $\mathrm{Ce}^{4+}$ in sol-gel dip-coating $\mathrm{SnO}_{2}$ thin films increases drastically its electrical resistivity. In the first case, it is due the acceptor-like nature of the doping ion, leading the matrix to high charge compensation. On the other hand, for $\mathrm{Ce}^{+4}$ doped samples, it is verified a broadening of the grain boundary depletion layer. Measurements under room pressure leads to higher intergrain potential barriers when compared to measurements carried out under vacuum conditions, due to oxygen adsorption at particles surface. The presence of $\mathrm{Ce}^{3+}$ increases the infrared transmittance, which means a lower free electron concentration. XANES data confirms that the thermal annealing at $550^{\circ} \mathrm{C}$ of thin films, although promotes oxidation to C $e^{4+}$, still keeps a significantly amount (about 60\%) of ions in the oxidation state C $e^{3+}$. Raman spectroscopy data show the evolution of the $\mathrm{SnO}_{2}$ bulk vibration modes with increasing thermal annealing temperature.

Keywords: tin dioxide, thin films, cerium, rare-earth, electrical transport.
\end{abstract}

\section{INTRODUÇÃO}

A produção de cerâmicas com propriedades eletrônicas que possam ser opticamente induzidas ou amplificadas, pode ter uma ampla aplicação na optoeletrônica. Dispositivos que possam ser opticamente integrados em sistemas de transmissão de dados necessitam de filmes finos com baixa perda óptica, dopado com elementos opticamente ativos tais como as terras-raras (RE). Estes íons possuem um vasto intervalo de transição, do ultravioleta ao infravermelho [1]. A combinação das transições de RE com semicondutores de gap largo, como é o caso do $\mathrm{SnO}_{2}(3,6 \mathrm{eV})$ [2] leva a uma alta eficiência na emissão [3, 4]. Além disso, $\mathrm{SnO}_{2}$ apresenta alta refletividade no infravermelho, transparência de cerca de $90 \%$ no visível $[5,6]$, e boa condutividade elétrica, propriedades que tornam este material adequado para várias aplicações tecnológicas, incluindo sensores de gás, células solares, entre outras [6,7]. O íon $\mathrm{Ce}^{3+}$ apresenta configuração eletrônica $4 \mathrm{f}^{1}$ no estado fundamental e $5 \mathrm{~d}^{1}$ no estado excitado. Emissão no azul tem sido reportada em torno de $489 \mathrm{~nm}$ [8]. Diferenças no raio iônico de $\mathrm{Ce}^{3+}$ e $\mathrm{Sn}^{4+}$ torna difícil a incorporação em $\mathrm{SnO}_{2}$ diminuindo a eficiência quântica no processo de luminescência [9]. A formação de nanopartículas pelo processo sol-gel tem sido usada para diminuir este problema [1, 4]. Porém, quando se obtém partículas de dimensões da ordem de 3 a $10 \mathrm{~nm}$ [10] na constituição de filmes finos, o transporte elétrico através da barreira de potencial no contorno de grão é dificultado, levando a um alto espalhamento de elétrons e conseqüentemente baixa mobilidade. Malagu e cols. [11] propuseram um modelo de barreiras Schottky em semicondutores do tipo-n nanoestruturados, que foi aplicado 
para $\mathrm{SnO}_{2}$ policristalino, composto de partículas menores que $10 \mathrm{~nm}$, fornecendo valores para a barreira Schottky, além de outros parâmetros importantes. Recentemente [12] concluímos que o mecanismo de condução Schottky [13] é fundamental em $\mathrm{SnO}_{2}$ dopado com Ce. As conclusões obtidas naquele trabalho, com relação ao transporte elétrico, estavam ligadas a que o tratamento térmico realizado nos filmes $\left(550^{\circ} \mathrm{C}\right)$ não alterasse significativamente o estado de oxidação do dopante.

Assim, este trabalho trata da verificação dos efeitos do tratamento térmico para a incorporação de Ce na matriz $\mathrm{SnO}_{2}$, com relação ao transporte elétrico e traz dados de espectroscopia Raman e análise via XANES (X-ray absorption near edge spectroscopy) usando radiação Síncrotron, que ajudam na interpretação dos mecanismos de transporte dominantes. O objetivo é contribuir para uma compreensão completa das características do transporte elétrico e, a médio prazo, colaborar para o desenvolvimento de dispositivos eletroluminescentes.

\section{MATERIAL E MÉTODO EXPERIMENTAL}

A suspensão coloidal obtida através do processo solgel envolve a dopagem de $\mathrm{SnO}_{2}$ com Ce, preparada através de uma solução aquosa de 0,5 molar de $\mathrm{SnCl}_{4} \cdot 5 \mathrm{H}_{2} \mathrm{O}$, adicionando-se a quantidade desejada de $\left(\mathrm{NH}_{4}\right)_{2} \mathrm{Ce}\left(\mathrm{NO}_{3}\right)_{6}$ ou $\mathrm{Ce}\left(\mathrm{NO}_{3}\right)_{3}$, obtendo-se assim uma dopagem com Ce ${ }^{4+}$ ou Ce $^{3+}$, respectivamente. Com isso foram obtidas soluções dopadas com $1 \%$ em mol de $\mathrm{Ce}^{4+}$ e dopadas com $1 \%$ e $0,1 \%$ em mol de $\mathrm{Ce}^{3+}$. Depois de adicionado o reagente dopante à solução aquosa em um béquer, sob constante agitação por meio de uma barra magnética, adiciona-se $\mathrm{NH}_{4} \mathrm{OH}$ (concentrado) até elevar o pH a 11. A suspensão sofre diálise contra água destilada por 10 dias para eliminação de íons $\mathrm{Cl}^{-} \mathrm{e} \mathrm{NH}_{4}^{+}$. A suspensão pronta para deposição de filmes apresenta pH 7. Os filmes finos são depositados via dip-coating, que consiste na imersão de um substrato em uma suspensão coloidal, a fim de formar uma fina camada do material de depósito. Essa imersão é feita controlando-se a velocidade, e em seguida, o substrato é retirado à mesma velocidade. Com o prosseguimento da secagem ou gelatinização, é formada uma camada sólida (filme). Desta forma o processo de dip-coating pode ser dividido em cinco estágios: imersão, emersão, deposição, evaporação e drenagem. A velocidade de imersão/emersão utilizada aqui foi de $10 \mathrm{~cm} / \mathrm{min}$ a temperatura de aquecimento entre camadas é de $400{ }^{\circ} \mathrm{C}$, por $10 \mathrm{~min}$ ao passo que a temperatura de tratamento térmico final, após 10 camadas é de $550^{\circ} \mathrm{C}$ por $1 \mathrm{~h}$.

Para as medidas de caracterização elétrica eletrodos de $\mathrm{Sn}$ foram evaporados por meio da técnica de evaporação resistiva em uma evaporadora Edwards Auto 306, em cadinhos de Mo e pressão da ordem de $10^{-6}$ torr. Após deposição através de uma máscara de sombra os contatos são submetidos a tratamento térmico a $180{ }^{\circ} \mathrm{C}$ por $30 \mathrm{~min}$. Experimentos de corrente em função da voltagem para diversas temperaturas e resistência em função da temperatura, seguiram dois procedimentos distintos: sob pressão ambiente, foram feitos em um forno EDG, e sob vácuo em um criostato com circuito fechado de gás He da Janis Research, acoplado com um controlador de temperatura da Lake Shore Cryotronics com $0,05 \mathrm{~K}$ de precisão.

Devido à baixa solubilidade dos íons $\mathrm{RE}$ em $\mathrm{SnO}_{2}$ $(0,05 \%)$, os experimentos de XANES foram realizados no modo de fluorescência. Os pós foram tratados a temperaturas distintas: $200,400,550$, e $1000{ }^{\circ} \mathrm{C}$ por $1 \mathrm{~h}$. Esses pós foram investigados por XANES, que foram feitas à temperatura ambiente, principalmente, no HASYLAB (HAmburg SYnchrotron radiation LABoratory) $(4,5 \mathrm{GeV}, 140 \mathrm{~mA}) \mathrm{em}$ Hamburgo, Alemanha. As medidas de XANES nas bordas $\mathrm{L}_{\text {III }}$ de Ce $(5723 \mathrm{eV})$, foram feitas nas estação E4, usando como monocromador um cristal duplo de Si (111). Os dados de XANES para filmes foram coletados no modo de detecção total de elétrons usando o detector desenvolvido por Tourillon et al. [14]

Dados de Raman foram obtidos usando um espectrofotômetro RXN1-785 KOSI, equipado com um diodo laser de infravermelho próximo (near-IR laser diode) com emissão de $785 \mathrm{~nm}$ (luz de excitação das amostras), e com um detector $\mathrm{CCD}$, para fornecer um espectro rápido e simultâneo dos dados de Raman de 100 a $3450 \mathrm{~cm}^{-1}$. Esses dados têm sua precisão garantida por uma rede de difração HoloPlex, KOSI, que fornece uma resolução espectral típica de $2 \mathrm{~cm}^{-1}$. A potência do laser na posição da amostra foi medida em um experimento separado, como sendo 30 a 50 $\mathrm{mW}$. Dados foram coletados com uma lente objetiva com uma ordem de ampliação de 10 vezes. Mais detalhes sobre o procedimento neste tipo de experimento foram publicados em outro lugar [15].

\section{RESULTADOS E DISCUSSÃO}

A Fig. 1 mostra dados de resistividade em função da temperatura para filmes finos de $\mathrm{SnO}_{2}$ dopados com Ce,

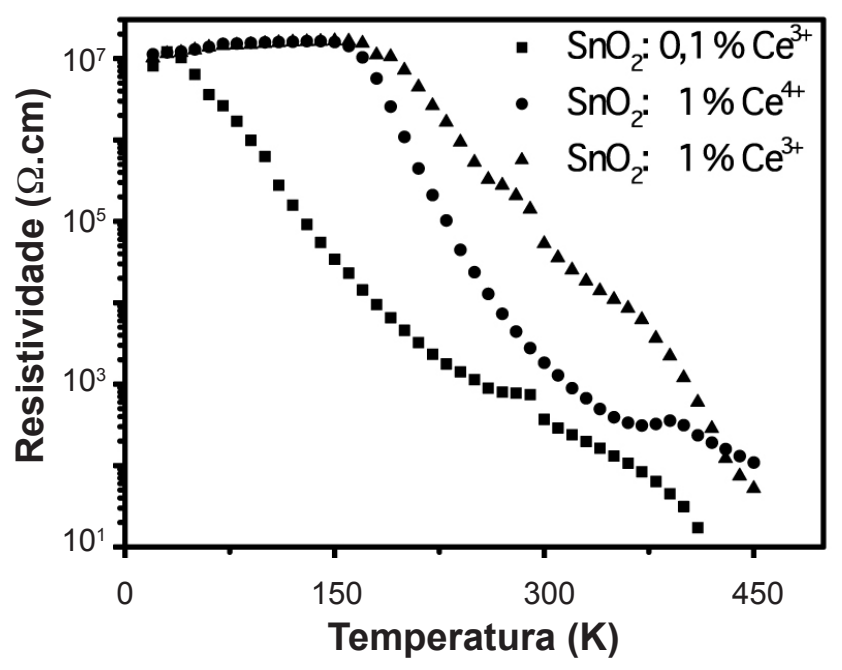

Figura 1: Resistividade elétrica em função da temperatura para filmes finos de $\mathrm{SnO}_{2}$ dopados com Ce, medidos sob pressão de $10^{-5}$ torr. [Figure 1: Electrical resistivity as a function of temperature for Cedoped $\mathrm{SnO}_{2}$ thin films, under pressure of $10^{-5}$ torr.] 
em diferentes concentrações e estados de oxidação, medidos sob pressão de $10^{-5}$ torr. A dopagem maior com $\mathrm{Ce}^{3+}$ leva a uma amostra claramente mais resistiva, o que sugere o comportamento aceitador do íon terra-rara na matriz, como seria de se esperar para uma substituição de $\mathrm{Sn}^{4+}$, já que a matriz é naturalmente do tipo $\mathrm{n}$ e, portanto, existe uma alta compensação de carga. Por outro lado, a amostra com dopagem de $\mathrm{Ce}^{4+}$ possui uma resistividade intermediária. Ainda que dados de solubilidade de $\mathrm{Ce}^{3+} \mathrm{em} \mathrm{SnO}_{2}$ não sejam conhecidos, sabe-se que para o $\mathrm{Eu}^{+3}$, este limite é da ordem de 0,05-0,06 at.\% [16]. O raio iônico é 0,69 Å para $S^{4+}$ e 0,947 para $\mathrm{Eu}^{3+}$ [17]. Como o raio iônico do $\mathrm{Ce}^{3+}$ é 1,01 $\AA$, espera-se uma solubilidade ainda menor para $\mathrm{Ce} \mathrm{em} \mathrm{SnO}_{2}$. Assim, ainda que uma porcentagem de íons $\mathrm{Ce}^{3+}$ possa estar em posição substitucional a $\mathrm{Sn}^{4+}$, não há duvidas que esta limitação do raio iônico leva também a uma contribuição efetiva de íons localizados no contorno das partículas. Portanto, ainda que o comportamento aceitador de $\mathrm{Ce}^{3+}$ na matriz seja um fator fundamental para a resistividade do filme, a baixa solubilidade contribui para um alto espalhamento no contorno de grão, o que justifica que a dopagem com $\mathrm{Ce}^{4+}$ também leve a uma alta resistividade, ainda que a substituição de $\mathrm{Sn}^{4+}$ por $\mathrm{Ce}^{4+}$ não leve a um comportamento aceitador.

Simulações pelo método de Rietveld [18] podem determinar a posição do íon dopante na matriz, ou seja, substitucional ou intersticial. Essa questão pode também ser analisada qualitativamente através dos valores obtidos para FWHM (largura da meia altura). No caso de substituições intersticiais, acontece uma diminuição no ordenamento de longo alcance, pois nesse caso, as substituições são aleatórias com referência as posições atômicas da matriz cristalina, nesse caso a estrutura rutilo, fase cassiterita; com isso teríamos uma diminuição na cristalinidade do material (aumento do FWHM). Já para substituição em posições da rede, esta só pode ocorrer num sítio de estanho, considerando ordens de longo alcance, o que proporcionaria um aumento no ordenamento de longa distância (diminuição do FWHM). Caso o dopante ocupasse o sítio do oxigênio, causaria mudanças de curto alcance e não seriam detectadas nos difratogramas. Dados publicados recentemente [12] mostram que há somente uma possibilidade que resulta em melhoria do ordenamento de longo alcance, ficando evidente a substituição. Com isso, a análise de Rietveld forneceu indicações de que uma porcentagem significativa do dopante ocupa o sítio esperado (Sn). No entanto, considerando a baixa solubilidade do terra-rara na matriz, como já discutido, esta análise tem que ser vista com ressalvas, pois a porcentagem de $\mathrm{Ce}^{3+}$ localizada no contorno de grão para uma dopagem da ordem de 1at\% é significativa e as condições de baixa simetria e ordem de curto alcance na região de depleção das partículas, não permitem definir um caráter substitucional para íons de Ce posicionados no contorno de grão.

A Fig. 2 mostra dados de transmitância no infravermelho próximo. Uma análise simples, baseada na teoria do gás de elétrons de Drude [19], confirma o caráter aceitador do $\mathrm{Ce}^{3+}$, pois a maior transmitância é para a maior dopagem, ou seja, para a menor concentração de elétrons livres.
Considerando que os filmes tenham a mesma espessura, o aumento da concentração do íon dopante $\mathrm{Ce}^{3+}$ aumenta a transmitância no infravermelho e, consequentemente, diminui a reflexão, pois induz maior compensação de carga, diminuindo a concentração de elétrons livres, em bom acordo com a teoria de Drude. Convém ressaltar que esse comportamento relacionado a um comprimento de onda de plasma baixo significa alta concentração de elétrons livres, da ordem de $10^{20} \mathrm{~cm}^{-3}$ [20]. Esse tipo de comportamento, ou seja, dependência das curvas de refletância e transmitância no infravermelho próximo, em função da concentração de íons dopantes, já foi observada anteriormente para amostras de $\mathrm{SnO}_{2}$ dopadas com $\mathrm{Sb}^{5+}$ [19-21]. Como naquele caso o íon dopante é um doador, o comportamento observado foi o inverso, ou seja, a maior concentração de $\mathrm{Sb}^{5+}$ leva a maior quantidade de elétron livres e conseqüentemente menor transmitância e maior reflexão no infravermelho próximo.

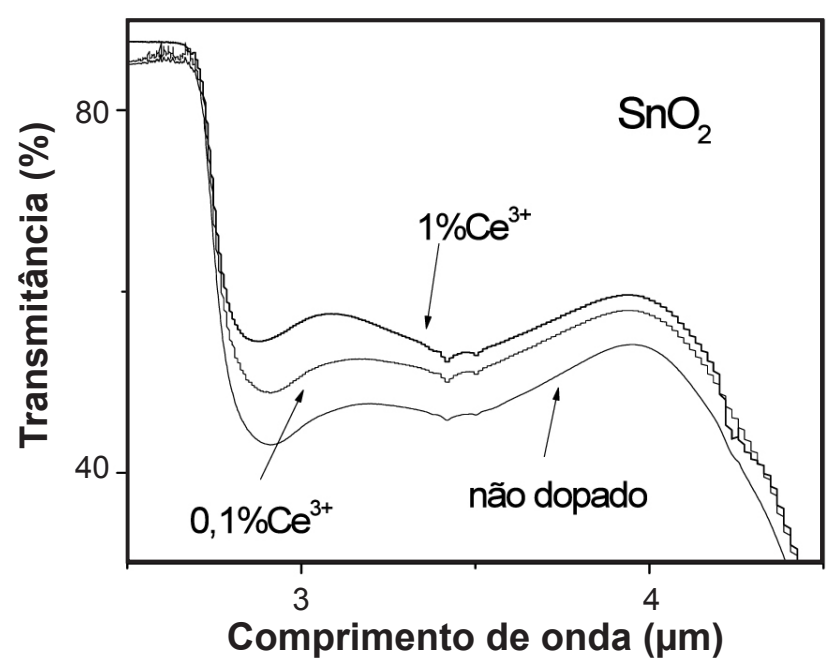

Figura 2: Transmitância no infravermelho próximo para filmes finos de $\mathrm{SnO}_{2}$ não dopado e dopados com $\mathrm{Ce}^{3+}$.

[Figure 2: Near infrared transmittance for undoped and $\mathrm{Ce}^{3+}$ doped $\mathrm{SnO}_{2}$ thin films.]

Medidas de corrente em função da voltagem (I x V), a diversas temperaturas podem ajudar na determinação dos tipos de mecanismos de transporte relevantes para o material analisado. Dentre esses mecanismos, a emissão termo-iônica Schottky é caracterizada por um decréscimo na barreira de potencial entre grãos devido a o campo elétrico aplicado, permitindo o movimento de elétrons através da barreira. A densidade de corrente (J) para este efeito é dada por [13]:

$$
\mathrm{J}_{\mathrm{S}}=\mathrm{A}^{*} \cdot \mathrm{T}^{2} \cdot \exp \left[-\left(\phi-\beta_{\mathrm{S}} \cdot \mathrm{E}^{1 / 2}\right) / \mathrm{kT}\right]
$$

na qual $\mathrm{A}^{*}$ é a constante de Richardson, $\beta_{\mathrm{S}}$ é o coeficiente Schottky, E é o campo elétrico aplicado e $\phi$ é a barreira de potencial entre grãos. Verifica-se facilmente que quando o transporte elétrico no material é dominado por este mecanismo, um gráfico de $\ln \mathrm{J}$ em função de $\mathrm{E}^{1 / 2}$, para $\mathrm{T}$ fixo, fornece uma relação linear. Deste ajuste, os parâmetros $\beta_{\mathrm{s}}$ e $\phi$ podem ser obtidos. 
A Fig. 3 representa um gráfico de (ln $\mathrm{J}$ ) em função da raiz quadrada do campo elétrico aplicado. A linha a-b representa o ponto onde a curva passa a ter comportamento linear. $\mathrm{O}$ campo elétrico necessário para que esse comportamento ocorra, diminui com a temperatura. A mudança na inclinação (c-d) é devido à eliminação de espécies $\mathrm{O}_{2}^{-}$, já que experimentos de dessorção de oxigênio a temperaturas programadas [22] indicam a eliminação desse íon em torno de $423 \mathrm{~K}\left(150{ }^{\circ} \mathrm{C}\right)$ para amostras de $\mathrm{SnO}_{2}$. Esse fenômeno seria responsável por um aumento abrupto na concentração de elétrons livres, levando a uma melhoria na condutividade da amostra, sendo representada pelo aumento significativo da densidade de corrente vista no gráfico. A porção linear dessas curvas, localizadas à direita da linha a-b, pode ser descrita por um fluxo de corrente no material, limitado pela taxa de elétrons excitados termicamente sobre a barreira de potencial no contorno de grão (emissão termiônica Schottky). $\mathrm{O}$ ajuste linear (feito com base na equação A) observado nas curvas da Fig. 3 é perfeito para todas as temperaturas, principalmente quando a eliminação de espécies $\mathrm{O}_{2}^{-}$não é

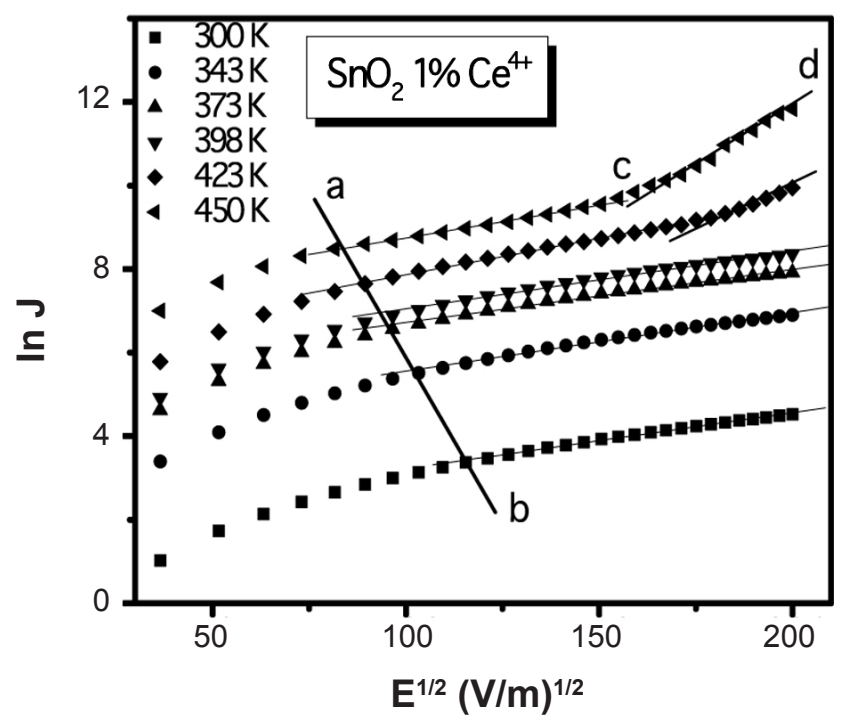

Figura 3: ln $\mathrm{J}^{\mathrm{x} \mathrm{E}^{1 / 2}}$ para $\mathrm{SnO}_{2}: 1$ at $\% \mathrm{Ce}^{4+}$, em várias temperaturas, pressão de $10^{-5}$ torr.

[Figure 3: $\ln \mathrm{J} \times E^{1 / 2}$ for $\mathrm{SnO}_{2}: 1 \mathrm{lat} \% \mathrm{Ce}^{4+}$, at several temperatures, under $10^{-5}$ torr.] observada [12]. Essa linearidade das curvas assegura que a emissão Schottky é o mecanismo dominante.

A análise completa da emissão Schottky para essas amostras já foi publicada [12] e a Tabela I é uma síntese dos parâmetros obtidos a partir do ajuste do mecanismo Schottky para situações diferentes de pressão, temperatura e estado de oxidação dominante do dopante introduzido.

Verifica-se na Tabela I que, em geral, $\phi$ apresenta uma variação pequena quando comparamos as amostras dopadas com $1 \% \mathrm{Ce}^{4+}$ e $1 \% \mathrm{Ce}^{3+}$ sob condições de vácuo. A concentração de elétrons livres é maior no filme com $1 \% \mathrm{Ce}^{4+}$, devido a esse dopante não apresentar comportamento aceitador (Fig. 1). Entretanto, o filme dopado com $\mathrm{Ce}^{4+}$ ainda apresenta uma alta resistividade, o que pode ser explicado pelos valores de $\beta_{\mathrm{s}}$ nesses filmes. Considerando que $\beta_{\mathrm{S}}$ é inversamente proporcional à largura da região de depleção [13], essa região de depleção no contorno de grão é maior para a amostra dopada com $\mathrm{Ce}^{4+}$ do que para aquela dopada com $\mathrm{Ce}^{3+}$. Embora a natureza do dopante seja a mesma, o que explica a mesma magnitude da barreira de potencial, o filme com dopante de maior estado de oxidação apresenta uma maior região de depleção no contorno de grão, que também leva a um maior espalhamento de elétrons e, consequentemente, uma menor mobilidade [12]. Embora a concentração de elétrons livres seja maior no filme de $\mathrm{SnO}_{2}: 1 \% \mathrm{Ce}^{4+}$, a resistividade desse filme ainda é alta, devido ao alto espalhamento de elétrons no contorno de grão. A Tabela I também permite fazer uma comparação entre medidas realizadas sob condições de baixa pressão (vácuo) e condições de pressão ambiente para a amostra de $\mathrm{SnO}_{2}: 1 \% \mathrm{Ce}^{4+}$. Comparando a altura da barreira de potencial $(\phi)$, observa-se que ela é maior para as medidas feitas na presença de oxigênio (pressão ambiente). Sob pressão ambiente existe uma maior adsorção de oxigênio nas regiões de superfície e contorno de grão do material, causando um acréscimo na altura da barreira Schottky. A dependência de $\phi$ e $\beta_{\mathrm{s}}$ com a temperatura já foi discutido em outro lugar [12] e está em bom acordo com os resultados obtidos por Malagu e cols. [11].

A Fig. 4 traz resultados de XANES para $\mathrm{SnO}_{2}$ dopado com $\mathrm{Ce}^{3+}$ submetido a diferentes temperaturas de tratamento térmico, por uma hora. Essa figura mostra um resultado

Tabela I - Estimativa da altura da barreira de potencial $(\phi)$ e coeficiente de emissão Schottky $\left(\beta_{\mathrm{S}}\right)$ para filmes finos SnO $:$ Ce. [Table I - Potential barrier $(\phi)$ and Schottky emission coefficient $\left(\beta_{S}\right)$ evaluation for $\mathrm{SnO}_{2}$ : Ce thin films.]

\begin{tabular}{|c|c|c|c|c|c|c|c|}
\hline \multicolumn{2}{|c|}{ Temperatura $(K)$} & 300 & 343 & 373 & 398 & 423 & 450 \\
\hline \multirow{2}{*}{$\begin{array}{c}\text { Vácuo } \\
\left(\text { pressão }=2 \times 10^{-5} \text { torr }\right) \\
1 \% C e^{3+}\end{array}$} & $\phi(e v)$ & 0,60 & 0,65 & 0,66 & 0,66 & 0,68 & 0,71 \\
\hline & $\beta_{S}\left(\mathrm{eVm}^{1 / 2} \mathrm{~V}^{1 / 2}\right) \times 10^{-4}$ & 3,62 & 4,24 & 5,04 & 5,80 & 7,33 & 8,96 \\
\hline \multirow{2}{*}{$\begin{array}{c}\text { Vácuo } \\
\left(\text { pressão }=2 \times 10^{-5} \text { torr }\right) \\
1 \% C e^{4+}\end{array}$} & $\phi(e V)$ & 0,58 & 0,60 & 0,62 & 0,66 & 0,68 & 0,69 \\
\hline & $\beta_{S}\left(\mathrm{eVm}^{1 / 2} \mathrm{~V}^{1 / 2}\right) \times 10^{-4}$ & 3,55 & 4,26 & 4,23 & 4,89 & 6,03 & 6,08 \\
\hline \multirow{2}{*}{$\begin{array}{c}\text { Pressão ambiente } \\
1 \% \mathrm{Ce}^{4+}\end{array}$} & $\phi(e V)$ & 0,61 & 0,67 & 0,71 & 0,75 & 0,74 & 0,80 \\
\hline & $\beta_{S}\left(\mathrm{eVm}^{1 / 2} \mathrm{~V}^{-1 / 2}\right) \times 10^{-4}$ & 3,47 & 3,88 & 4,47 & 5,41 & 6,87 & 12,3 \\
\hline
\end{tabular}




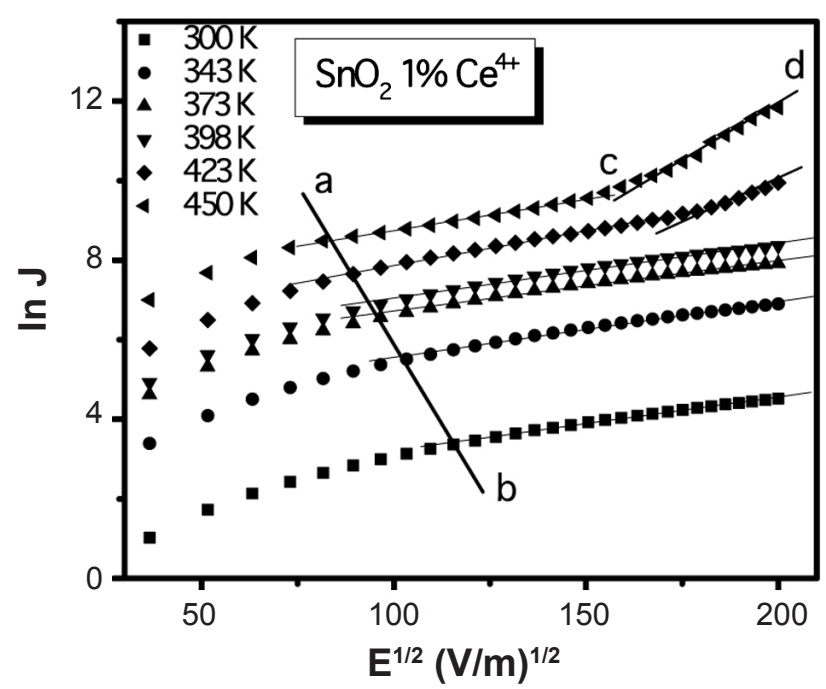

Figura 4: Espectro de XANES de $\mathrm{SnO}_{2}$ dopado com $\mathrm{Ce}^{3+}$ com temperaturas de tratamento térmico variado.

[Figure 4: XANES spectra for $\mathrm{Ce}^{3+}$ doped $\mathrm{SnO}_{2}$ submitted to several distinct thermal annealing temperatures.]

bastante interessante associado aos estados de oxidação deste dopante, o que é fundamental para o entendimento dos mecanismos dominantes de transporte elétrico [12]. Conforme a temperatura de tratamento térmico aumenta, o espectro se torna mais achatado e um novo pico se torna mais evidente. Os picos relevantes neste caso são: $5727 \mathrm{eV}$ (linha branca do $\mathrm{Ce}^{3+}$ ) e $5738 \mathrm{eV}\left(\right.$ do $\mathrm{Ce}^{4+}$ ) [23]. O aumento da banda em $5738 \mathrm{eV}$ evidencia a oxidação do íon $\mathrm{Ce}^{3+}$ para $\mathrm{Ce}^{4+}$ com o aumento da temperatura de tratamento térmico. No entanto, mesmo após o tratamento térmico a $1000{ }^{\circ} \mathrm{C}$, a proporção $\mathrm{Ce}^{3+} / \mathrm{Ce}^{4+}$ é de aproximadamente $60 / 40$. Este valor precisa ser confirmado por um cálculo mais exato, usando como padrão uma amostra de $\mathrm{SnO}_{2}: \mathrm{Ce}^{4+}$, o que já está sendo feito, devendo ser publicado oportunamente. No entanto, a estimativa feita é bem razoável e sua implicação é que no caso do tratamento térmico de filmes a $550^{\circ} \mathrm{C}$, a proporção de $\mathrm{Ce}^{3+}$ é ainda maior, e que portanto, este estado de oxidação deste íon é dominante no material. Assim, como este íon tem um comportamento aceitador na rede de $\mathrm{SnO}_{2}$, explica-se facilmente a resistividade elétrica mais alta, conforme mostrado na Fig. 1, em boa concordância com a maior transmitância no infravermelho (Fig. 2).

Resultados de espectroscopia Raman para uma região do espectro de interesse $\left(350\right.$ a $\left.850 \mathrm{~cm}^{-1}\right)$ estão na Fig. 5, para pós de $\mathrm{SnO}_{2}$ dopados com $1 \% \mathrm{Ce}^{3+}$ tratados a diferentes temperaturas de tratamento térmico por $1 \mathrm{~h}$. Para a amostra sem tratamento térmico (como preparada) os modos de vibração a $570 \mathrm{~cm}^{-1}$ e a $500 \mathrm{~cm}^{-1}$ são evidentes e estão relacionados a picos de desordem estrutural $\mathrm{S}_{1}$ e $\mathrm{S}_{2}$, característicos de nanopartículas [24]. Para tratamento térmico a $200{ }^{\circ} \mathrm{C}$, começam a aparecer alguns picos característicos de vibração de ligações na rede cristalina de $\mathrm{SnO}_{2}$, tais como o $\mathrm{A}_{1 \mathrm{~g}}$, a $640 \mathrm{~cm}^{-1}$. A medida que a temperatura de tratamento térmico cresce, aparecem outros picos em torno de $480 \mathrm{~cm}^{-1} \mathrm{e} 780 \mathrm{~cm}^{-1}$, também relacionados à vibração $\mathrm{Sn}-\mathrm{O}[25]$ ou seja, $\mathrm{E}_{\mathrm{g}}$ e $\mathrm{B}_{2 \mathrm{~g}}$, modos ópticos de vibração de $\mathrm{SnO}_{2}$ [24]. Esses picos ficam bem evidentes quando a temperatura de tratamento térmico é $1000{ }^{\circ} \mathrm{C}$, o que está associado ao tamanho do cristalito que cresce com a temperatura. Este tipo de análise fornece uma característica importante para o transporte elétrico neste material, ou seja, ainda que os pequenos cristalitos sugiram que os modos de espalhamento no bulk não são importantes, o aparecimento dos picos Raman associados a vibração do bulk contradizem esta hipótese. Assim, a análise completa do comportamento elétrico deve levar em conta que a existência de partículas nanoscópicas implica num predomínio do espalhamento de elétrons no contorno e grão, porém os modos de espalhamento no bulk, tais como impurezas ionizadas e vibrações da rede, podem se tornar importantes, dependendo da temperatura ou concentração de impurezas (dopagem) introduzidas na matriz de $\mathrm{SnO}_{2}$.

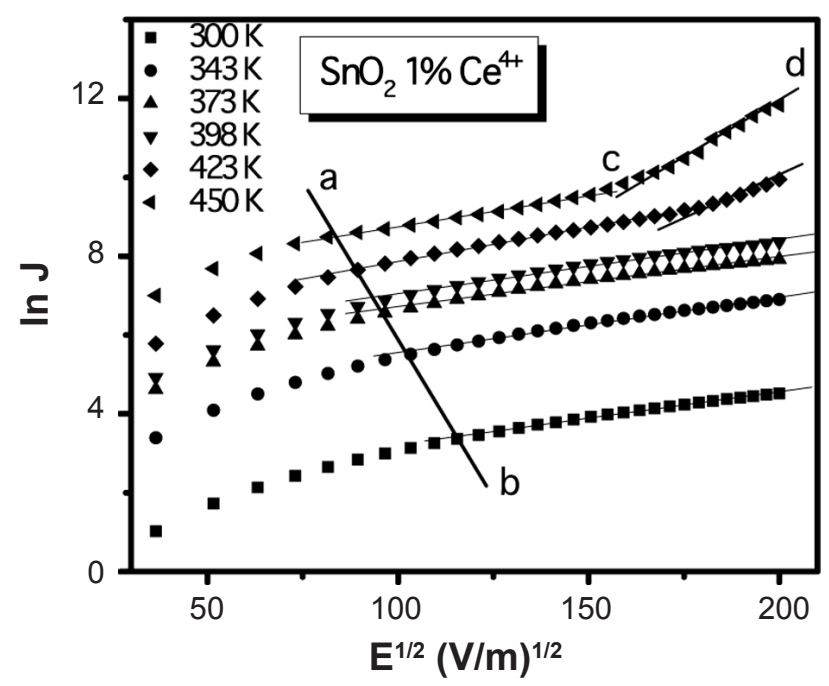

Figura 5: Espectroscopia Raman de $\mathrm{SnO}_{2}$ dopado com $\mathrm{Ce}^{3+}$ com diferentes temperaturas de tratamento térmico.

[Figure 5: Raman spectroscopy of $\mathrm{Ce}^{3+}$-doped $\mathrm{SnO}_{2}$ submitted to several thermal annealing temperatures]

\section{CONCLUSÃO}

A incorporação de $\mathrm{Ce}$ em $\mathrm{SnO}_{2}$ aumenta drasticamente a resistividade elétrica. Se o estado de oxidação do dopante for $\mathrm{Ce}^{3+} \mathrm{o}$ aumento é atribuído ao comportamento aceitador do dopante, substitucional a $\mathrm{Sn}^{4+}$. Por outro lado a dopagem com $\mathrm{Ce}^{4+}$ também leva a uma alta resistividade, o que provavelmente está relacionado ao aumento da região de depleção no contorno de grão, conforme avaliado a partir do modelo Schottky de condução termoiônica para a barreira intergranular. A maior transmitância óptica no infravermelho de filmes com maiores concentrações de $\mathrm{Ce}^{3+}$ é um resultado em concordância com a maior resistividade elétrica, causada pelo comportamento aceitador, com alta compensação de carga na matriz. A linearidade da relação corrente voltagem para temperaturas fixas permite a avaliação da barreira de 
potencial intergranular e permite verificar a contribuição das espécies de oxigênio adsorvidas. Sob condições de vácuo um mecanismo de liberação de espécies $\mathrm{O}_{2}^{-}$tornase evidente acima de $150{ }^{\circ} \mathrm{C}$. Resultados de XANES para pós de $\mathrm{SnO}_{2}$ dopados com Ce mostram a evolução da concentração de $\mathrm{Ce}^{4+}$ conforme a temperatura de tratamento térmico de $\mathrm{SnO}_{2}: \mathrm{Ce}^{+3}$ aumenta, porém mesmo a $1000{ }^{\circ} \mathrm{C}$ ainda existe uma proporção significativa de $\mathrm{Ce}^{3+}$, de modo que isso também deve ocorrer nos filmes tratados a $550{ }^{\circ} \mathrm{C}$. Assim sendo as conclusões referentes ao comportamento elétrico dos filmes devem ser baseadas no comportamento aceitador de $\mathrm{Ce}^{3+}$, considerando o predomínio deste estado de oxidação na matriz. A compreensão dos mecanismos de transporte elétrico neste material deve contribuir para um melhor conhecimento em direção ao desenvolvimento de dispositivos eletroluminescentes.

\section{AGRADECIMENTOS}

Os autores agradecem as agências de fomento: CAPES, CNPq e FAPESP e também a PROPe-UNESP, convênio Santander, pelo auxilio recebido por L. V. A. Scalvi para estágio no Lab. SOLEIL, França.

\section{REFERÊNCIAS}

[1] F. Gu, S. F. Wang, M. K. Lu, Y. X. Qi, G. J. Zhou, J. Cryst. Growth 255 (2003) 357.

[2] S. C. Ray, M. K. Karanjai, D. Dasgupta, Surf. Coat. Technol. 102 (1998) 73.

[3] M. Nogami, T. Enomoto, T. Hayakawa, J. Lumin. 97 (2002) 147.

[4] F. Gu, S. F. Wang, M. K. Lu, Y. X. Qi, Opt. Mater. 25 (2004) 59

[5] E. Dien, J. M. Laurent, A. Smith, J. Eur. Ceram. Soc. 19 (1999) 787.

[6] A. C. Yanes, J. Del Castillo, M. Torres, J. Peraza, V. D. Rodriguez, J. Mendes-Ramos, Appl. Phys. Lett. 85 (2004) 2343.
[7] J. Rockenbecher, U. zum Felde, M. Tischer, L. Troger, M. Haase, H. Weller, J. Chem. Phys. 112 (2000) 4296.

[8] K. Annapurna, R. N. Dwivedi, P. Kundu, S. Buddhydu, Mater. Lett. 58 (2004) 787.

[9] H. Zhang, X. Fu, S. Niu, G. Sun, Q. Xin, J. Lumin. 115 (2005) 7.

[10] E. A. Morais, L. V. A. Scalvi, S. J. L. Ribeiro, V. Geraldo, Phys. Stat. Solidi a 202 (2005) 301.

[11] C. Malagù, V. Guidi, M. Stefancich, M. C. Carotta, G. Martinelli, J. Appl. Phys. 91 (2002) 808.

[12] M. A. L. Pinheiro, T. F. Pineiz, E. A. Morais, L. V. A. Scalvi, M. J. Saeki, A. A. Cavalheiro, Thin Solid Films 517 (2008) 976.

[13] J. G. Simmons, J. Phys. D 4 (1971) 613.

[14] G. Tourillon, E. Dartyge, A. Fontaine, M. Lemonnier, F. Bartol, Phys. Lett. A 121 (1987) 251.

[15] V. Briois, D. Vantelon, F. Villain, B. Couzinet, A.-M. Flanka, P. Lagarde, J. Synchrotron Rad. 14 (2007) 403.

[16] T. Matsuoka, T. Tohda, T. Nitta, J. Electrochem. Soc. 130 (1983) 417.

[17] R. D. Shannon, Acta Crystallographica A 32 (1976) 751.

[18] H. M. Rietveld, J. Appl. Crystallogr. 10 (1969) 65.

[19] E. Santhi, V. Dutta, A. Banerjee, K.L. Chopra, J. Appl. Phys 51 (1980) 6243.

[20] V. Geraldo, L. V. A. Scalvi, P.N. Lisboa-Filho, C. M. Santos, J. Phys. Chem. Solids 67 (2006) 1410.

[21] V. Geraldo, L. V. A. Scalvi, E. A. Morais, C. V. Santilli, S. H. Pulcinelli, Mater. Res. 6 (2003) 451.

[22] N. Yamazoe, J. Fuchigami, M. Kishikawa, Surface Sci. 86 (1979) 335.

[23] V. Briois, D. Lutzenkirchen-Hecht, F. Villain, E. Fonda, S. Belin, B. Griesebock, R. Frahm, J. Phys. Chem. A 109 (2005) 320

[24] A. Diéguez, A. Romano-Rodríguez, A. Vila, J. R. Morante, J. Appl. Phys. 90 (2001) 1550

[25] P. S. Patil, R. K. Kawar, T. Seth, D. P. Amalnerkar, P. S. Chigare, Ceram. Int. 29 (2003) 725.

(Rec. 19/11/2009, Ac. 01/06/2010) 